\title{
Mycobacteriosis in the Compromised Host
}

\author{
PH Lagrange ${ }^{+}$, A Wargnier, JL Herrmann
}

Service de Microbiologie, Hôpital Saint-Louis, Assistance Publique-Hôpitaux de Paris, Université Paris VII, 1 Avenue Claude Vellefaux, 75475 Cedex 10 Paris, France

The studies of rare genetic defects, the preliminary results of population-based studies, being validated by the experimental immunocompromised animal models and the current observations accumulated in immunocompromised patients with mycobacterial diseases provide us with insights into the importance of the macrophage activation pathway in controlling human infection with pathogenic and non pathogenic intracellular multiplying mycobacteria.

Initial cytokine production by infected macrophages and/or dendritic cells could be crucial in the overall regulation of self cure, acquired protection or immunopathological sequelae expressing the disease. Knowledge of molecular and genetic cross-talks between phagocytic and specialized antigen presenting cells and different mycobacterial products associated with persistence or replication of the intracellular bacteria, could provide further informations on the global immune regulation of the early host responses to infection and the following events. It seems likely that the development of mycobacterial infections in humans will turn out to be as much dependent on the genetic make up of the host as or the virulence of the bacteria.

Key words: tuberculosis - acquired immunodeficiency syndrome-Aids - atypical mycobacteria - knockout mouse

There is a long history of the association of opportunistic mycobacterial diseases with the immunocompromised hosts. Individual risk factors for tuberculosis (TB) with conditions such as measles, Hodgkin's disease, and corticosteroid immune suppression given during organ transplantation were well recognised. However, other groups of immunocompromised patients are also at risk of TB and mycobacteria other than tuberculosis (MOTT) infections. Recent reports have described several groups of patients with acute leukemia, lymphoma, visceral malignancies and treated with immunosuppressive therapy who have been infected with Mycobacterium tuberculosis and others MOTT such as $M$. avium, $M$. fortuitum, $M$. chelonae, M. scrofulaceum and M. hemophilum (Skogberg et al. 1993, Young 1996). However, it was not until the post-human immunodeficency virus (HIV) era, that renewed interest became widespread in mycobacteriosis (mycobacterial diseases) in the immunocompromised host. Several reasons for such interest are the following. First, re-emergence of TB in countries where TB was in the way of eradication, was puzzling. This being also associ-

\footnotetext{
${ }^{+}$Corresponding author. Fax: +33-14-249.9200

E-mail : phl@chu-stlouis.fr

Received 7 August 2000

Accepted 4 September 2000
}

ated with nosocomial outbreaks, spreading by individuals with acquired immunodeficiency syndrome (Aids) carrying multiple-drug resistant (MDR) strains to others immunocompromised HIV patients but also to normal hosts such as healthcare workers in hospitals or in the community, was frightful. Second, MOTT that were generally quite rarely isolated before the advent of Aids, but represented before the recently used efficient antiretroviral therapies, a considerable role in morbidity and mortality. This was particularly true of the M. avium complex (MAC) because these organisms are the single most important cause of disseminated bacterial infection in Aids patients (Benson 1994). Third reason, a newly recognised pediatric syndrome, the idiopathic mycobacterial infection in non immuno-compromised children (Levin et al. 1995), led to the identification of mendelian susceptibility to mycobacterial infection and several specific genetic deficiencies including receptors genes for important cytokines such as IFN $\gamma$ and IL-12 (interleukin) have been described (Jouanguy et al. 1999).

\section{NATURAL HISTORIES OF MYCOBACTERIAL DISEASES}

Among the mycobacterial genus, the vast majority of species are saprophytic belonging to the environmental microflora. They are present at different latitudes, and could be isolated from soil, and water. There are only a limited number of pathogenic mycobacteria for men, as shown in the Table. The natural history of pathological and casual opportunist mycobacteria diseases differ due 
to different tissue tropisms: the MOTT opportunists appear to be more limited than M. tuberculosis or M. leprae in parallel with the experimental observations of more limited virulence. For instance, experimental infection with $M$. tuberculosis is often lethal in normal non-immuno-compromised mice, in contrast virulent strains of $M$. avium infection are only lethal in immunodeficient mice (Collins 1972). Moreover, among the M. avium group, the serovars 1 and 2 have a definite pathogenicity for certain species of domestic animals (Thoen 1994).

It should be noted that the most common area of involvement for MOTT after the respiratory tract, is the gastrointestinal tract which occurred after colonisation of the mucosal epithelium followed by endocytosis within mucosal lymphoepithelial sites (Lugton 1999). There is also in vitro evidence that mycobacteria can adhere to and penetrate epithelial cells other than M cells of the Peyer's patches. Several human epithelial cell lines are permissive to invasion by $M$. avium, $M$. tuberculosis and other rapidly growing mycobacteria. Local immune non specific or specific defect of the mucosa-associated lymphoid tissues (MALT) could be associated with loss of tolerance to the resident mycobacteria and the establishment of a local granuloma formation and necrosis. Only severe and prolonged immunodepression is associated with atypical mycobacterial dissemination.

In contrast, airborne infection of the lower respiratory tract of humans, cattle or small laboratory animals with $M$. tuberculosis, has been extensively studied and well described, showing the direct involvement of alveolar macrophages as portal of entry of such pathogenic mycobacterial species (Hopewell 1988). Acquisition of tubercle bacilli through the oropharynx and gastrointestinal tract by ingesting contaminated food or milk is much less common. From 3\% to $10 \%$ of newly infected persons will develop clinically apparent TB. In the other 90 to $97 \%$, host defences are sufficient to prevent progression from infection to disease. Cellmediated immunity is the most important defense mechanism and it contributes in controlling the infection by killing many organisms and walling off the remainder within immune granulomas. When immunity waves, as by the effects of age, or is suppressed, either by the influence of drugs or diseases, the potential exist for either the reactivation at wherever endogenous sites of dormant persisting viable tubercle bacilli or for the development of newly acquired TB from recently inhaled bacilli from an exogenous source. Undoubtedly, both forms occur and the likelihood of one or the other will depend on the background prevalence of TB in the immunocompromised host group and the risk of contracting active cases of TB in the community.

Worlwide, TB is the most common opportunistic bacterial infection in patients with HIV co-infection. Before the efficient antiretroviral therapies, in developed countries, disseminated MAC disease was the most common systemic bacterial opportunistic infection in patients with Aids. Both contribute substantially to morbidity and mortality in this population. The risk factors, clinical syndromes and development in the investigations, prevention and treatment are discussed in the first part in this review. The second part is devoted to the acquisition of knowledge of immune defences in experimentally and transgenic immuno-compromised mouse models. And finally the third part is reporting new data concerning Mendelian susceptibility to mycobacterial infection in men.

\section{MYCOBACTERIAL DISEASES IN HIV COINFECTED PATIENTS}

Since the first publications of increased numbers of disseminated life-threatening infection with M. avium in 1982 by Greene et al. (1982) and increased prevalence of tuberculosis in patients

TABLE

Mycobacteria species isolated more frequently in microbiology laboratories

\begin{tabular}{lllll}
\hline Growers & Highly pathogens & Pathogenic & $\begin{array}{l}\text { Casual pathogens } \\
\text { (opportunist) }\end{array}$ & Saprophytic \\
\hline Slow & M. tuberculosis & M. ulcerans & M. avium & M. gordonae \\
& M. bovis & M. malmoense & M. intracellulare & M. terrae \\
& M. africanum & M. szulgai & M. scrofulaceum & M. nonchromogenicum \\
& M. leprae & M. simiae & M. kansasii & M. flavescens \\
& M. hemophilum & M. asiaticum & M. xenopi & M. gastri \\
& & & M. triviale \\
Fast & & M. marinum & \\
& & M. chelonae & M. snegmatis \\
& & M. fortuitum & \\
\hline
\end{tabular}


with Aids in 1984 by Pitchenik et al. (1984), several reviews has been published concerning the infections with pathological and opportunistic mycobacteria in HIV coinfected patients. Many of them involved on progress made in defining those at risk, characterising pathogen virulence factors, the host immune responses, development of rapid and sensitive diagnostic tools and therapeutic guidelines. However, natural pathogenic and atypical mycobacteria differ in their relationships with the immunocompromised host. Concerning the natural highly pathogens in immunocompetent hosts, i.e. M. tuberculosis and M. leprae, only the former was associated with an increased risk for developing the disease in HIV coinfected patients. By contrast, in countries where leprosy is a local endemic disease, no publication is reporting any increase in the prevalence of the disease as such or of any increase of the lepromatous leprosy forms. Such difference in the morbidity between these two pathogens might be explained through different virulence factors, one of them being the very low replicating rate of M. leprae in vivo and as such a quite longer incubation period varying from 10 to 15 years in the infected person. Concerning the atypical MOTT, the great majority of species isolated was M. avium, and this was observed mainly in developed countries, in contrast to what was reported in Africa, for instance, where such species are very rarely isolated in Aids patients.

\section{TUBERCULOSIS AND HIV COINFECTION}

Infection with HIV increases the risk of development or clinical TB, either as a result of reactivation of after primary exposure. The emergence and rapid development of the HIV/Aids pandemic has had a devastating impact on the global burden of TB and is now by far the most important predisposing factor for active TB in those individuals infected by the tubercle bacillus.

Clinical presentation and radiological findings are directly dependent upon the level of immunosuppression (Halvir \& Barnes 1999). For instance, mycobacteremia and extrapulmonary TB become more common in patients with low CD4 counts. In HIV coinfected patients with TB, who have CD4 cell counts of 200 or more per $\mathrm{mm}^{3}$, chest radiographic findings include upper lobe infiltrates and cavitation, similar to those in HIV negative TB patients. In HIV TB patients with fewer than 200 CD4/ $\mathrm{mm}^{3}$, mediastinal adenopathy is common (similar to that in HIV-negative patients with primary TB). It should be noted also that approximately $5 \%$ of HIV patients with pulmonary TB have positive results on acid-fast straining of sputum, despite normal chest radiographs.

Usually skin testing with 5 TU of PPD is the method by which TB infection is identified. All HIVinfected persons with a positive tuberculin skin test result equal or larger than $5 \mathrm{mn}$ should undergo chest radiography and clinical evaluation in order to exclude active $\mathrm{TB}$, and should receive recommended chemoprophylaxis.

Clinical studies have shown the detrimental effect of TB on the course of HIV infection. The risk of death in HIV-TB patients was reported to be twice than in HIV infected patients without TB, independently of the CD4 counts (Whalen et al. 1995). Such higher mortality rate appeared to be due to progressive HIV infection rather than TB, and the degree of immunosuppression being the most important predictor of survival of HIV-TB patients. M. tuberculosis probably increases HIV replication by inducing macrophages to produce transactivating cytokines (TNF $\alpha$, IL-1, IL-6). New efficient anti retroviral combination regimens associated with anti-TB chemotherapy have shown a dramatic improvement for the pronostic in HIVTB patients who are infected with drug susceptible tubercle bacillus. Moreover, adjunctive treatments aiming at limiting the hyperproduction of $\mathrm{TNF} \alpha$, such as thalidomide has been used in controlled trials in order to minimise the HIV trasnsactivation during TB (Haslett et al. 1999).

In HIV-infected patients with drug susceptible $\mathrm{TB}$, the standard six month regimen results in prompt sterilisation of sputum, similar to those in HIV-negative TB patients. However, several studies showed higher rates of relapse in HIV-TB patients who received 6 as compared with 9 to 12 months of anti-TB therapy.

The actual guidelines of the Centers for Diseases Control (USA) state that the minimum duration of therapy is 6 months, but if clinical and bacteriological response is slow, treatment should be given for a total period of 9 months, or 4 months after the culture become negative.

MDR TB have been responsible of explosive nosocomial outbreaks in several cities of the world with an unprecedented case fatality rate of $80 \%$ in HIV-infected patients. Stringent infection-control policies curtailed these outbreaks in New York city and Miami and a strengthened public health infrastructure and widespread use of directly observed therapy (DOT) resulting in a $44 \%$ reduction in MDR TB in New York between 19911994. Recommendations on the allocation of adequate public health resources to ensure that every patient complete their anti-TB therapy is urgently needed in countries where prevalences of MDR TB and HIV endemic are growing. Clinicians should strongly consider measuring drugs levels to evaluate the possibility of malabsorption in case 
of no response to anti TB therapy in HIV-infected patients who adhere correctly to it.

HIV-infected patients with recent or remote TB infection are at extremely high risk for the development of active TB disease, and the efficience of chemoprophylaxis has been demonstrated. Isoniazid (INH) for 6 months reduced the risk of TB by approximately $70 \%$ in HIV-positive patients with positive PPD skin test. Preliminary data suggest that the efficacy of daily rifampin and pyrazinamide for 2 months is similar to 12 months of INH (Rose 1998). Because HIV-infected patients have defective cellmediated immunity, and those with a CD $4 / \mathrm{mm}^{3}$ lower than 200 , have quite often false negative tuberculin skin tests, it has been recommended that skin testing be performed with PPD and other antigens to detect anergy. However, the great variability of anergic responses and the fact that chemoprophylaxis with INH does not reduce the incidence of TB in $\mathrm{HIV}$-infected persons with anergy, anergy testing is no longer recommended to assess the risk of TB infection. An ongoing study is performed in our laboratory to evaluate a new surrogate marker for infection. It consist of testing for specific IgG antibody detection against $M$. tuberculosis glycolipids in HIV infected patients at risk of TB, since it has be shown that such antibodies were present in serum of HIVTB patients, long before the clinical diagnostic of TB (Simonney et al. 1995).

TB is often the initial clinical manifestation of HIV coinfection, and all patients with TB should be tested for HIV because of the potential benefits of an early diagnosis of HIV infection. Also, all patients with recently diagnosed HIV infection should be skin tested with tuberculin.

Highly active antiretroviral therapy (HAART) employing both the protease inhibitor (PI) class of drug and the nonnucleoside reverse transcriptase inhibitors has come into wide care among HIV-infected individuals, and this HAART might impact in two ways on TB treatment in Aids patients. The first one is related to the risk of substantial drug interactions between the PI antiretroviral and the rifamycins. The second involved the development of paradoxical reactions during anti-TB therapy. Such paradoxical worsening of the disease develop in up to $36 \%$ of these patients, characterised by fever, peripheral and mediastinal lymphadenopathy or worsening chest infiltrates or radiography (Narita et al. 1998). Such reactions suggest reactive inflammation from a stronger immune response to M. tuberculosis during effective antiretroviral therapy. Usually self-limited, these reactions generally last 10 to 40 days, but in case of severe reactions, they may require a short course of treatment with glucocorticoid (Narita et al. 1998).
Immunomodulating agents have been tested in order, either to compensate deficiency or to modulate excessive cytokine production. Recently, Condos et al. (1997) reported some success on the administration of IFN $\gamma$ via aerosol to five patients, with refractory MDR-TB, one patient was HIV coinfected. Thalidomide has recently been given in a group of TB patients being HIV-non infected and HIV-infected. Levels of IFN $\gamma$ increased, stimulated $\mathrm{TNF} \alpha$ production decreased and weight gain was enhanced during thalidomide treatment (Tramontana et al. 1995). No follow-up was reported concerning the viral load and pronostic of HIV infected patients. At least, immunotherapy using an injection of a killed suspension of a nonpathogenic environmental mycobacteria $(M$. vaccae) has been evaluated in several trials with some beneficial effects. However, the last study done in South Africa (Durban Immunotherapy Trial Group 1999) showed that M. vaccae immunotherapy has no benefit when added to standard anti-TB chemotherapy.

\section{ATYPICAL MYCOBACTERIA AND HIV COINFEC- TION}

Previously a rare cause of pneumonia in patients with pre-existing lung disease, MAC was recognised early in the Aids pandemic as a cause of serious disseminated infection (Greene et al. 1982) and was the most common cause of systemic bacterial infection in Aids, affecting more than $50 \%$ of patients in the developed countries. Primary prophylaxis was recommended on the basis of $\mathrm{CD}^{+}$cell threshold (less than $50 / \mathrm{mm}^{3}$ ) in all HIV persons for increased risks of specific opportunistic infections, such as MAC disease. However, more potent antiretroviral regimens have been introduced recently that suppress HIV replication and increase the $\mathrm{CD} 4^{+}$cell counts, and marked decrease in the incidence of major infectious complications, especially MAC disease, have been observed everywhere in patients treated with these regimens (Palella et al. 1998).

Of the species composing MAC, $M$. avium is most commonly associated with disease in Aids, and serotypes 1, 4 and 8 are the most commonly isolated from symptomatic patients with Aids (French et al. 1997). Furthermore, epidemiological studies suggest that MAC strains associated with pulmonary disease may differ from those associated with disseminated disease in Aids patients. Moreover, phylogenic analysis of nucleotide sequences data, using the variable 16S-23 rDNA internal transcribed spaces (ITS) in clinical isolates from Aids patients showed that the disseminated disease-associated MAC strains were distinct by ITS sequence analysis from other isolates 
(Frothingham \& Wilson 1994). Lastly, two particular epidemiological facts are intriguing. First, simultaneous infection of Aids patients with more than one strain appears to be rather common (Arbeit et al. 1993). Second, even if the prevalence of disseminated MAC associated disease was high, great individual susceptibility, geographic and seasonal variations has been described. For instance, disseminated MAC is rare in Africa, although MAC is prevalent in soil and water samples from the area where advanced Aids patients are present (Morrisey et al. 1992). These phenomenon remains unexplained, although it is postulated that widespread previous antimycobacterial immunity from the high rate exposure of Africans to M. tuberculosis and BCG vaccination may be responsible. This is supported by data which suggest that prior TB diagnosis may be somehow protective against disseminated MAC (Horsburgh et al. 1996).

The pathogenesis of MAC infection is incompletely understood. Disseminated MAC is believed usually to follow primary acquisition of the mycobacteria. It appears that MAC first colonizes the gastrointestinal (GI) tract or respiratory mucosa, and dissemination follows. Several animal models have shown that dissemination occurs after colonisation of the GI tract. Epidemiological studies in humans did confirm the preceding isolation of MAC from stool or sputum within one year before bacteremia in $60 \%$ of patients with $\mathrm{CD}^{+}$cell counts less than $50 \mathrm{CD} 4 / \mathrm{mm}^{3}$. Individual susceptibility to disseminated MAC disease could be due also to some additive non specific incompetence to kill or to slow down mycobacterial replication. Such hypothesis is reinforced by recent findings showing higher serum levels of both $\mathrm{TNF} \alpha$ and soluble TNF $\alpha$ receptors in $M$. avium complex disseminated disease in Aids patients, which is directly correlated with the degree of 1,25 dihydroxyvitamin D3 deficiency (Hang et al. 1996).

In contrast to the immunocompetent host, in which MAC disease is usually limited to the lungs, in patients with Aids, bacteremia is by far the most common syndrome. Frequently, there is wide spread dissemination, often to bone marrow, liver, lymph nodes and spleen. When the clinical syndrome suggests disseminated MAC, mycobacterial cultures of the peripheral blood should be obtained and are often positive. As shown in lepromatous leprosy, if histologic sections are obtained by tissue biopsies, they showed aggregates of foamy histiocytes, in which the organisms can be seen by acid fast staining. Such positive biopsies may be positive before blood cultures.

Several studies have reported negative predictors for survival of Aids patients with disseminated MAC disease: the $\mathrm{CD}^{+}{ }^{+}$cell counts, and the initial higher level of mycobacteremia (Horsburgh et al. 1994). Moreover, there are also clinical and abnormal laboratory values which are associated with MAC bacteremia, thus being useful to manage the patients in whom this infection is suspected and should be confirmed by blood culture. Such abnormal findings among patients with less $50 \mathrm{CD} 4$ cells/ $\mu$ are: the documentation of fever on more than 30 days during the preceding 3 months, a previous Pneumocystis carinii treated infection, an hematocrit of less than $30 \%$, or a serum albumin concentration of less than $3 \mathrm{~g} / \mathrm{dl}$ (Chin et al. 1994).

It has been clearly shown that the survival of Aids patients with disseminated MAC disease is directly related to the most efficient antimycobacterial chemotherapy given. Curative therapy should include rifabutin, a new macrolide (clarithromycine or azithromycine) and ethambutol. However, designing the ideal therapeutic regimen for disseminated MAC is complicated by the lack of clinically usefull susceptibility testing for most of the available agents. Only clarithromycine susceptibilities have been shown to correlate clinical efficacy. MAC cocultivation within macrophages (Sison et al. 1996a) or MAC infected beige (C57 BL/6/bg/bg) mice gave better clinical correlations (Sison et al. 1996b).

Current guidelines for MAC prevention include the use of rifabutin. In light of current results concerning the new macrolides, clarithromycine or azithromycine, alone or the combination of a macrolide and rifabutin are well considered. Discontinuation of prophylaxis should also be considered if the CD4+ cell counts rise above 100/ $\mu \mathrm{l}$ after efficient antiretroviral therapy.

Immune modulators, such as granulocyte-macrophage-colony stimulating factor (GMCSF), granulocyte-colony-stimulating factor (G-CSF), interferon $\gamma$ and IL-2 may be of benefit in enhancing intracellular killing of M. avium (Benson 1995).

\section{IMMUNODEFICIENCY AS A SOURCE OF KNOW- LEDGE}

Immunological deprivation, using chemotherapeutic regimens or monoclonal antibodies on the susceptibility of animals (mosly done in mice) to mycobacterial disease due to $M$. bovis $\mathrm{BCG}$ or to virulent $M$. tuberculosis have shown that the expression of optimal antimycobacterial immunity requires the coordinated activity of a whole range of T-cell and macrophage functions, including those of TCR $\alpha \beta^{+}$CD4 and CD8 T cells (Kaufmann 1993). The results from studies with $M$. tuberculosis infection has suggested a major protective role for INF $\gamma$, TNF $\alpha$, IL-12 and inductible nitric oxide synthase (INOS). The use of gene-disrupted mice has 
led to new insights into how several immunological effectors protect mice against mycobacterial infections (Cooper et al. 1997). The data from these knockout mice showed that IFN $\gamma$ and TNF $\alpha$ are the primary mediators of macrophage activation and sterilizing granuloma formation. At least, a primary mechanism of mycobacterial control is the production of nitric oxide (NO) by INOS. As innate production of INF $\gamma$ is insufficient to control totally M. tuberculosis in mice, expansion of INF $\gamma$ production is needed. The cells considered responsible for such expansion has been shown to be $\mathrm{CD}^{+}$and $\mathrm{CD}^{+}$lymphocytes. The expression of IFN $\gamma$-producing $\mathrm{T}$ lymphocytes in these animal models has been shown to be dependent upon the early production of IL-12. Although the $\mathrm{CD} 4^{+} \mathrm{T}$ lymphocytes is implicated as the primary protective $\mathrm{T}$ cell subset, the $\mathrm{CD} 8^{+}$lymphocytes has been also implied later during the infection. However, the absence of any difference between control mice and mice lacking in the common lytic mechanisms (such as granzyme B and perforine) seems to indicate that their role during the first phase of infection is mediated more by the cytokine production than by lysis of infected cells. The putative role of $\mathrm{T}$ cell has been highlighted in controlling early aspects of monocytes migration and in aiding maturation of granuloma.

In conclusion, immunodeficient gene-disrupted mice have already contributed a great deal to our knowledge of the nature of immunity to $M$. tuberculosis. Thus, in experimental mycobacterial infection in mice, lack of any of the above mentioned defense mechanisms cannot be fully compensated for by alternative immune mechanisms. These mechanisms appear to have a cascade effect on each other, leading to the generation of an optimal response. However, if the broad components of human antimycobacterial immunity are similar with those of mice, several significant differences in details exist: regulation of NO synthesis, macrophage killing M. tuberculosis induced by IFN $\gamma$, and the gene coding for the natural resistance associated macrophage protein (NRAMP1) (Kumararatne 1997). Thus it is important to consider not only the acquired immunodeficiency in mice, but also the natural defects associated with higher susceptibility to mycobacterial diseases in humans, in order to better understand the immunological basis of resistance to these diseases.

Recent studies have been shown selective susceptibility to non classical pathogenic mycobacteria, such as environmental MOTT and $B C G$ vaccine. The selective immunodeficiency has been suspected to be a mendelian disorder, and recent studies were undertaken to demonstrate its molecular basis.
By contrast with the well-known leading causes of disseminated disease in Aids patients, the MOTT and BCG vaccine have been shown also to cause severe disease in otherwise healthy children without any over immunodeficiency (Levin et al. 1995, Casanova et al. 1995, 1996). Unlike severe immunocompromised patients, being highly susceptible to a broad range of opportunistic pathogens, children with disseminated idiopathic BCG and MOTT infections do not have generally associated infections, apart from salmonellosis in less than half of the cases. In the cases of children with the idiopathic disseminated BCG infection, a mendelian disorder of autosomal recessive inheritance was suggested by the high rates of affected siblings and parental consanguinity, together with the equal number of male and female children (Casanova et al. 1996). The same observations were also reported in the Levin's study (Levin et al. 1995). Moreover, the heterogeneity of clinical outcome and its correlation with the histopathological granulomatous type of the lesions, suggested that the underlying potential inherited disorder might be genetically heterogeneous (Jouanguy et al. 1996).

Following a whole genome search, the first gene was mapped to the region of chromosome $6 \mathrm{a}$ containing the IFN $\gamma$ receptor ligand-binding chain gene (R1) and single base substitution in the coding region of this gene, resulting in a new stop codon, and in the complete absence of IFN $\gamma$ receptor expression on cell surface was identified first in London (Newport et al. 1996). Concurrently, Casanova and collaborators in Paris, sequencing the IFN $\gamma \mathrm{R} 1$ gene in a consanguineous Tunisian family, affected by disseminated BCG infection, identified a frameshift deletion that resulted in the absence of IFN $\gamma$ R1 (Jouanguy et al. 1999).

Since these two initial reports concerning the IFN $\gamma$ R1 gene, as a cause of the unique susceptibility to atypical mycobacterial infections in childhood, several other mutations in this gene have been identified that were associated with the complete absence of IFN $\gamma$ receptor expression on cell surface in patients with severe clinical disease often fatal. The identification of IFN $\gamma$ R1 deficiency as a cause of susceptibility to mycobacterial infection in humans confirmed indirectly the validity of using knockout mouse model to identify key pathways controlling susceptibility and resistance to these bacteria. Moreover, human equivalents of the murine defects in other key cytokines or their receptors controlling macrophage activation were also identified. For instance, mutations in the IL-12 gene or its receptor were also shown to be associated with susceptibility to disseminated BCG or MOTT infection and do cause clinical syndromes 
similar, but milder, to that associated with the IFN $\gamma$ R1 deficiency (Jouanguy et al. 1999). Furthermore, mutations in the second signaling chain of the IFN $\gamma$ receptor (IFN $\gamma$ R2) have been identified in a child with disseminated M. avium and M. fortuitum infection (Dorman \& Holland 1998).

It seems very likely that single gene defects in the different components of the cascade of events involving macrophage activation pathway in humans will be ultimately identified. However, such selective susceptibility associated with gene defects are not representing all the possibilities involved in susceptibility to classical pathogens such as M. tuberculosis and M. leprae, since higher incidence of $\mathrm{TB}$, or disseminated $\mathrm{TB}$ and lepromatous leprosy have not been reported in the preceding described patients.

Individual susceptibility to develop the diseases, such or TB or leprosy, have been suscepted for long time to be associated with genetic factors (Jepson 1998), and functional polymorphims in gene controlling macrophage activation might have an higher numerical implication than gene defects associated with selective susceptibility to atypical mycobacteria or BCG vaccine. The question of functional polymorphims in major genes controlling mycobacterial replication in the host are being assessed in different population-based studies. Some interesting data have been reported concerning the NRAMP gene (Bellamy et al. 1998), and the results obtained with the Belem Family study (Blackwell et al. 1997) are indicative of several putative candidate genes/regions identified through analysis of disease susceptibility phenotypes in murine models of infectious disease as a lead to identification of susceptibility genes in humans.

\section{REFERENCES}

Arbeit RD, Sluskey A, Barber TN, et al. 1993. Genetic diversity among strains of Mycobacterium avium causing monoclonal and polyclonal bacteremia in patients with AIDS. J Infect Dis 167: 1384-1390.

Bellamy R, Ruwende C, Corrah T, et al. 1998. Variations in the NRAMP1 gene and susceptibility to tuberculosis in west africans. $N$ Engl J Med 338: 640-644.

Benson C 1994. Mycobacterium tuberculosis and Mycobacterium avium complex disease in patients with HIV infection. Curr Op Infect Dis 7: 95-105.

Benson CA 1995. Mycobacterium avium complex disease in patients with AIDS. Infect Dis Clin Pract 1: 1-15.

Blackwell JM, Black GF, Peacock CS, et al. 1997. Immunogenetics of leishmanial and mycobacterial infections: the Belem Family study. Phil Trans $R$ Soc London B 352: 1331-1345.

Casanova JL, Emile JF, Blanche S, et al. 1996. Idiopathic disseminated BCG infection: a french national retrospective study. Pediatrics 98: 774-778.
Casanova JL, Jouangy E, Lamhamedi S, Blanche S, Fischer A 1995. Immunological conditions of children with disseminated BCG infection. Lancet 346 : 581.

Chin DP, Reingold AL, Horsburgh CR, et al. 1994. Predicting Mycobacterium avium complex bacteremia in patients infected with human immunodeficiency virus: a prospectively validated model. Clin Infect Dis 19: 668-674.

Collins F 1972. Acquired resistance to mycobacterial infection. Adv Tuberc Res 18: 1-30.

Condos R, Rom WN, Schluger NN 1997. Treatment of multidrug-resistant pulmonary tuberculosis with Interferon-gamma via aerosol. Lancet 349: 15131515.

Cooper AM, Saunders BM, D'Souza CD, Frank AA, Orme IM 1997. Mycobacterium tuberculosis driven processes in gene-disrupted mice. Bull Inst Pasteur 95: 85-96.

Dorman SE, Holland SM 1998. Mutation in the signaltransducing chain of the interferon-gamma receptor and susceptibility to mycobacterial infection. J Clin Invest 101: 2364-2369.

Durban Immunotherapy Trial Group 1999. Immunotherapy with Mycobacterium vaccae in patients with newly diagnosed pulmonary tuberculosis: a randomized controlled trial. Lancet 354: 116-119.

French AL, Benator DA, Gordin FM 1997. Non tuberculous mycobacterial infections. Management of HIV-infected patient, part II. Med Clinics North America 81: 361-379.

Frothingham R, Wilson KH 1994. Molecular phylogeny of Mycobacterium avium complex demonstrates clinically meaningful divisions. J Infect Dis 169: 305312.

Greene JB, Sidhu GS, Lewin S, et al. 1982. Mycobacterium avium-intracellulare: a cause of disseminated life-threatening infection in homosexual and drug abusers. Ann Inter Med 97: 539-546.

Halvir DV, Barnes PF 1999. Tuberculosis in patients with human immunodeficiency virus infection. $\mathrm{NEngl}$ J Med 340: 367-373.

Hang CJ, Aukrust P, Lien, et al. 1996. Disseminated Mycobacterium avium infection in AIDS: immunopathogenic significance of an activated tumor necrosis factor system and depressed serum levels of 1,25 Dihydrovitamin D. J Infect Dis 173: 259-262.

Haslett PAJ, Klausner JR, Makonkawkeyoon S, et al. 1999. Thalidomide stimulates $T$ cell responses and interleukin -12 production in HIV-infected patients. AIDS Res Hum Retrovir 15: 1169-1179.

Hopewell PC 1988. Mycobacterial diseases. In JF Murray, JA Nadel, Text Book of Respiratory Medicine, WB Saunders Co, Philadelphia, p. 856-915.

Horsburgh CR, Hanson DL, Jones JL, Thompson SE III 1996. Protection from M. avium complex disease in human immunodeficiency virus-infected patients with a history of tuberculosis. J Infect Dis 174: 1212-1217.

Horsburgh CR, Metchock B, Gordon SM, et al. 1994. Predictors of survival in patients with AIDS and dissseminated Mycobacterium avium complex dis- 
ease. J Infect Dis 170: 573-577.

Jepson A 1998. Twin studies for the analysis of heritability of infectious diseases. Bull Inst Pasteur 96: 71-81.

Jouanguy E, Altare F, Lamhamedi S, et al. 1996. Interferon- $\alpha$-receptor deficiency in an infant with fatal Bacille Calmette Guerin infection. N Engl J Med 335: 1956-1964.

Jouanguy E, Döffinger R, Dupuis S, Pallier A, Altare F, Casanova JL 1999. IL-12 and INF $\gamma$ in host defense against mycobacteria and salmonella in mice and men. Curr Op Immunol 11: 346-351.

Kaufmann SHE 1993. Immunity to intracellular bacteria. Annu Rev Immunol 11: 129-163.

Kumararatne DS 1997. Tuberculosis and immunodeficiency of mice and men. Clin Exp Immunol 107: 11-14.

Levin M, Newport MJ, D’Souza S, et al. 1995. Familial disseminated atypical mycobacterial infections in childhood: a human mycobacterial susceptibility gene? Lancet $345: 79-83$.

Lugton IW 1999. Mucosa-associated lymphoid tissues as sites for uptake, carriage and excretion of tubercle bacilli and other pathogenic mycobacteria. Immunol Cell Biol 77: 364-372

Morrisey AB, Aiku T, Falkinham JO, et al. 1992. Absence of Mycobacterium avium complex disease in patients with AIDS in Uganda. J AIDS 5: 477-478.

Narita M, Askin D, Hollender FS, Pitchenik AE 1998. Paradoxical worsening of tuberculosis following antiretroviral therapy in patients with AIDS. Am J Resp Crit Care Med 158: 157-161.

Newport M, Huxley CM, Huston S, et al. 1996. A mutation in the interferon-gamma receptor gene and susceptibility to mycobacterial infection. $N$ Engl J Med 335: 1941-1949.

Palella Jr FJ, Delany KM, Moorman AC, et al. 1998. Declining morbidity and mortality among patients with advanced human immunodeficiency virus infection. N Engl J Med 338: 853-860.

Pitchenik AE, Cole C, Russell BW, et al. 1984. Tubercu- losis, atypical mycobacteriosis, and the acquired immunodeficiency syndrome among Haïtian and non Haïtian patients in South Florida. Ann Inter Med 101: 641-645.

Rose DN 1998. Short course prophylaxis against tuberculosis in HIV-infected persons. A decision and costeffectiveness analysis. Ann Intern Med 129: 779 786.

Simonney N, Molina JM, Molinard M, Oksenhendler E, Perronne C, Lagrange PH 1995. Analysis of the immunological humoral response to Mycobacterium tuberculosis glycolipid antigens (DAT, PGLTb1) for diagnostic of tuberculosis in HIV-seropositive and seronegative patients. Eur J Microbiol Infect Dis 14: 883-891.

Sison JP, Yao Y, Kemper CA, et al. 1996. Treament of Myco-bacterium avium complex infection: does the Beige mouse model predict therapeutic outcome in humans? J Infect Dis 173: 750-753.

Sison JP, Yao Y, Kemper CA, et al. 1996. Treatment of Mycobacterium avium complex infection: do the results of in vitro susceptibility test predict therapeutic outcome in humans? J Infect Dis 173: 677-683.

Skogberg K, Ruutu P, Tukiainen P, Valtonen 1993. Effect of immunosuppressive therapy on the clinical presentation and outcome with tuberculosis. Clin Infect Dis 17: 1012-1017.

Thoen CO 1994. Mycobacterium avium infections in animals. Res Microbiol 145: 173-177.

Tramontana JM, Utaipat U, Molloy A, et al. 1995. Thalidomide treatment reduce tumor necrosis factor alpha production and enhances weight gain in patients with pulmonary tuberculosis. Mol Med 1: 384-397.

Whalen C, Horsburgh CR, Horn D, et al. 1995. Accelerated course of human immunodeficiency virus infection after tuberculosis. Am J Resp Crit Care Med 151: $129-135$.

Young LS 1996. Mycobacterial infections in the immunocompromised host. Curr Op Infect Diseases 9: 240-245. 